\title{
Effects of Genipin Concentration on Cross-Linked Chitosan Scaffolds for Bone Tissue Engineering: Structural Characterization and Evidence of Biocompatibility Features
}

\author{
Simona Dimida, ${ }^{1}$ Amilcare Barca, ${ }^{2}$ Nadia Cancelli, ${ }^{1}$ Vincenzo De Benedictis, \\ Maria Grazia Raucci, ${ }^{3}$ and Christian Demitri ${ }^{1}$ \\ ${ }^{1}$ Department of Engineering for Innovation, University of Salento, Lecce, Italy \\ ${ }^{2}$ General Physiology Laboratory, Department of Biological and Environmental Sciences and Technologies, \\ University of Salento, Lecce, Italy \\ ${ }^{3}$ Institute of Polymers, Composites and Biomaterials (IPCB), National Research Council of Italy, \\ Mostra d'Oltremare, Pad. 20, Viale Kennedy 54, 80125 Naples, Italy \\ Correspondence should be addressed to Christian Demitri; christian.demitri@unisalento.it
}

Received 8 February 2017; Accepted 6 March 2017; Published 13 April 2017

Academic Editor: Nobuhiro Kawatsuki

Copyright (C) 2017 Simona Dimida et al. This is an open access article distributed under the Creative Commons Attribution License, which permits unrestricted use, distribution, and reproduction in any medium, provided the original work is properly cited.

Genipin (GN) is a natural molecule extracted from the fruit of Gardenia jasminoides Ellis according to modern microbiological processes. Genipin is considered as a favorable cross-linking agent due to its low cytotoxicity compared to widely used cross-linkers; it cross-links compounds with primary amine groups such as proteins, collagen, and chitosan. Chitosan is a biocompatible polymer that is currently studied in bone tissue engineering for its capacity to promote growth and mineral-rich matrix deposition by osteoblasts in culture. In this work, two genipin cross-linked chitosan scaffolds for bone repair and regeneration were prepared with different GN concentrations, and their chemical, physical, and biological properties were explored. Scanning electron microscopy and mechanical tests revealed that nonremarkable changes in morphology, porosity, and mechanical strength of scaffolds are induced by increasing the cross-linking degree. Also, the degradation rate was shown to decrease while increasing the crosslinking degree, with the high cross-linking density of the scaffold disabling the hydrolysis activity. Finally, basic biocompatibility was investigated in vitro, by evaluating proliferation of two human-derived cell lines, namely, the MG63 (human immortalized osteosarcoma) and the hMSCs (human mesenchymal stem cells), as suitable cell models for bone tissue engineering applications of biomaterials.

\section{Introduction}

The occurrence of bone defects with critical size impairing regeneration and self-repair is a key problem in orthopaedic surgery [1]. As a result of a serious accident, trauma, deep burns, osteomyelitis, necrosis, bone tumours, and several other conditions, it is often necessary to remove the diseased tissue or bone fragments that have no vascularization and cannot be used for reconstructive surgery. Therefore, the resulting large bone defects have to be stabilized and/or reconstructed, by adopting bone substitution techniques and materials.

In most cases, the ideal solution is the autologous bone graft, which is the gold standard in clinical practice, because it possesses all the characteristics for bone growth: osteoconductivity, osteoinductivity, and osteogenicity [1-3]. Although it is a safe solution for compatibility and for the absence of immunogenicity, autograft is affected by problems such as donor site morbidity and limited supply [4]. Bone allografts and xenografts are alternatives, but they are expensive and imply the risk of disease transmission and adverse host immune reaction.

Synthetic bone substitutes with autogenous cell transplantation might overcome the aforementioned issues. Several materials have been developed and analyzed to be used for this purpose, including bioactive ceramics such as hydroxyapatite (HA) [5], beta-tricalcium phosphate (b-TCP) [6], biphasic calcium phosphate (BCP) [7], calcium phosphate 
cements [8], bioactive glass [9], and several biodegradable polymers [10-13].

Bioactive ceramics are chemically similar to natural bone inorganic components which allow osteogenesis to occur and can provide bony contact or bonds with the host bone [14]. However, brittleness and low biodegradability restrict their clinical applications. In this respect, natural and synthetic polymers could overcome these drawbacks. In particular, chitosan (CS) has recently attracted much interest in bone tissue engineering for its capacity to promote growth and mineral-rich matrix deposition by osteoblasts in culture [15]. In addition, CS is biocompatible, biodegradable, nontoxic, and physiologically inert and can be moulded into porous structures [16].

Chitosan is a natural copolymer composed of $\beta-(1-4)$ linked 2-acetamido-2-deoxy-D-glucopyranose and 2-amino2-deoxy-D-glucopyranose units, derived from the alkaline deacetylation of chitin, the main constituent of the exoskeleton of crustaceans, such as shrimps, molluscs, and insects [16-18]. It is also the principal fibrillar polymer in the cell wall of certain fungi. Chitin is one of the most abundant organic materials, being second only to cellulose in the amount produced annually by biosynthesis, and it is composed of $\beta$-(1-4)-linked 2-acetamido-2-deoxy-D-glucopyranose units. The chemical $\mathrm{N}$-deacetylation of chitin is achieved by alkaline hydrolysis and produces 40 to $80 \%$ of deacetylated CS [19].

The deacetylation degree (DD) of CS influences its solubility in aqueous solutions or its chemical modifications. In fact, the presence of reactive amino side groups provides many possibilities of chemical bonds and crosslinking reactions; commonly, to stabilize CS hydrogels and to enhance their mechanical properties and biodegradability and/or to ameliorate cellular adhesion properties, chemical or ionic cross-linking methods (e.g., UV, ionic cross-linking) are employed [20]. For instance, chemical cross-linkers such as epoxy compounds [21], aldehydes (formaldehyde, glyceraldehyde, and glutaraldehyde) [22], and carbodiimides [23] or ionic cross-linkers such as sulfates, citrates, and phosphates $[24,25]$ have been used to stabilize CS [17].

This paper is focused on the preparation and characterization of an innovative CS scaffold produced starting from chitosan hydrogel covalently cross-linked with genipin, a natural extract of Gardenia jasminoides Ellis [26]. Genipin has emerged as a favorable cross-linking agent due to its low cytotoxicity compared to the aforementioned widely used cross-linkers [27]. Genipin cross-links primary amine groups, and it has been extensively investigated in crosslinking both 2D gels and 3D scaffolds fabricated using aminecontaining polymers such as CS, collagen, and gelatin [26]. In a previous work, the polymerization reaction of a CS/genipin system, induced in the presence of oxygen radicals, was investigated. The reaction was strongly affected by temperature and by the presence of $\mathrm{H}^{+}$[28]. The aim of this research is to assess the effect of genipin on morphology, degradation, and mechanical properties of CS/genipin scaffolds and to describe the potential applications in bone tissue engineering by assessing cellular responses. For this purpose, two genipin concentrations were selected, namely, GN1 and GN2 (GN2 concentration is double that of GN1), for CS cross-linking.
Then, by adopting a human osteoblast-like immortalized cell line (MG63) and a human mesenchymal stem cell line (hMSC), the basic evaluation of biocompatibility and proliferation parameters was described.

\section{Materials and Methods}

2.1. Materials. Chitosan (CS) powder (average Mw: $75 \%$ DD, CAS 9012-76-4) and acetic acid (purity 98\%) were purchased from Sigma-Aldrich, and genipin (purity 98\%, HPLC grade) was purchased from Wako Chemicals Inc., USA. Lysozyme for degradation assays was purchased from Sigma-Aldrich (CAS 12650-88-3), and phosphate-buffered saline (PBS) solution was from Fluka. All products were used without any further purification. For the cell culturebased experiments, growth media, sera, supplements, and chemical reagents, such as 3-(4,5-dimethylthiazol-2-yl)-2,5diphenyltetrazolium bromide (MTT), were purchased from Sigma-Aldrich; and standard cell culture plastic materials (including disposable pipettes and tips) were used.

\subsection{Methods}

2.2.1. Preparation of Genipin-Chitosan Scaffolds. Chitosan (CS) membranes were prepared by the freeze-drying method. First, $1.5 \mathrm{~g}$ of CS was suspended in $100 \mathrm{~mL}$ of $0.1 \mathrm{M}$ acetic acid solution in a glass flask and stirred for $6 \mathrm{~h}$ at $4^{\circ} \mathrm{C}$ until complete dissolution. Afterwards, genipin was added to the solution and stirred for another hour. Batches with different genipin concentrations were prepared in order to obtain samples with different degrees of cross-linking and properties. In particular, two different concentrations of genipin were used: $3.75 \% \mathrm{w} / \mathrm{w}$ of dry chitosan $(0.25 \mathrm{M}$ final concentration) and $7.5 \% \mathrm{w} / \mathrm{w}$ of dry chitosan $(0.50 \mathrm{M}$ final concentration), called GN1 and GN2, respectively. Solutions were poured into Petri dishes with $60 \mathrm{~mm}$ diameter $(10 \mathrm{~g}$ of solution/dish) and thermally treated in an oven at $37^{\circ} \mathrm{C}$ for $24 \mathrm{hrs}$ and then freeze-dried for $21 \mathrm{~h}$. Scaffolds with $6 \mathrm{~mm}$ diameter and $3.7 \mathrm{~mm}$ thickness were obtained from the membranes cut by means of a surgical punch (biopsy punch, Kai Medical BB-807).

2.2.2. Scanning Electron Microscopy (SEM). GN1 and GN2 scaffolds materials were analyzed in vertical and transversal cross sections by scanning electron microscopy (Zeiss EVO 40) in low-vacuum modality and by applying a voltage of $25 \mathrm{kV}$. Samples were placed on the SEM sample holder using double-sided adhesive tapes and were observed without any further manipulation. Images were acquired (60x and 300x magnification) in order to visualize both the gross structure and the fine pore structure of each scaffold typology.

2.2.3. In Vitro Degradation Test. Each cylindrical sample $(6 \mathrm{~mm} \times 4 \mathrm{~mm})$ was weighed $\left(W_{0}\right)$ and immersed in $15 \mathrm{~mL}$ of PBS solution ( $\mathrm{pH} 7.4)$. After incubation at $37^{\circ} \mathrm{C}$, at every time point $(7,14,21$, and 28 days), samples were taken from the medium, washed in distilled water to remove buffer salts, and dried in an oven at $45^{\circ} \mathrm{C}$ for $12 \mathrm{hrs}$ under vacuum to remove 
excess water before freeze-drying. The extent of the in vitro degradation was calculated as the percentage of weight loss:

$$
\text { Weight loss }(\%)=100 \times\left\{\frac{\left(W_{0}-W_{t}\right)}{W_{0}}\right\} \text {, }
$$

where $W_{t}$ and $W_{0}$ are the final and the initial weights of the scaffold, respectively. These tests were performed simultaneously in PBS supplemented with lysozyme (0.05\%), a specific enzyme that hydrolyzes the $\beta$-(1-4) glycosidic linkage of chitosan backbone. This condition should mirror the scaffold behavior in the presence of degradation processes due to the metabolic activity of cultured cells. All the assays were repeated with four independent sample replicates.

2.2.4. Compression Test. Compression tests of GN1 and GN2 scaffolds were performed in wet conditions using PBS solutions by means of a universal testing machine until $75 \%$ deformation $(10 \mathrm{~N}$ load cell at a displacement rate $=$ $0.01 \mathrm{~mm} / \mathrm{s}$ ). The samples were hydrated in PBS solution at room temperature for $1 \mathrm{~h}$ prior to testing. During the compression test, the load and displacement were monitored and recorded. Five samples from each group (GN1 and GN2) were tested to obtain average and standard deviation value of elastic modulus $\left(E_{\text {low }}\right)$ calculated by initial slope of the stressstrain curves.

2.2.5. Contact Angle. The hydrophilic nature of genipin crosslinked chitosan hydrogels at the abovementioned genipin concentrations (3.75 and $7.5 \%$ genipin) was measured with a sessile drop water contact angle measurement (Krüss, DSA100, Germany) at room temperature. A non-cross-linked chitosan hydrogel has been used as a control.

A small drop of water was deposited onto the surface of gels and the contact angle was measured within $5 \mathrm{~s}$. The contact angle values reported were the averages of three consecutive measurements for each sample.

2.2.6. Fourier Transform Infrared (FTIR) Spectroscopy Analysis. FTIR spectra were recorded on a Nexus FTIR (Nicolet Spectrometer) equipped with an attenuated total reflectance (ATR, Smart ARK, Nicolet) crystal sampler. Film samples were used directly on an ATR crystal sampler at a resolution of $4 \mathrm{~cm}^{-1}$, average of 64 scans, at an absorbance range from 4000 to $400 \mathrm{~cm}^{-1}$.

\subsubsection{Cell Culture Conditions and Treatments}

Cytocompatibility Assays on the MG63 Human OsteoblastLike Cell Line. Analyses of cytocompatibility in vitro were performed by adopting the MG63 cell line (human osteosarcoma); MG63 were maintained in sterile conditions, in $75 \mathrm{~cm}^{2}$ plastic flasks, with Eagle's minimum essential medium (E-MEM) supplemented with $10 \%$ (v/v) fetal bovine serum (FBS), $2 \mathrm{mM}$ L-glutamine, $100 \mathrm{U} / \mathrm{mL}$ penicillin, and $100 \mathrm{ng} / \mathrm{mL}$ streptomycin, in a water-saturated atmosphere of $5 \% \mathrm{CO}_{2}$ and $95 \%$ air $\left(37^{\circ} \mathrm{C}\right)$. For propagation, cells at
70-90\% confluence (every 2-3 days) were washed twice in D-PBS (Dulbecco's PBS) before detaching with a $0.3 \%(\mathrm{v} / \mathrm{v})$ trypsin solution and harvesting by centrifugation; then, cell pellets were resuspended and transferred to new flasks. All experiments were performed between passages 3 and 10 of propagation. For the assessment of cytotoxicity, MG63 cells were seeded onto scaffolds in 24 -well plates $\left(5 \times 10^{4}\right.$ cells/scaffold) and incubated for $6 \mathrm{~h}$ to allow adhesion; then, a growth medium was added to cover completely the scaffold surface, and cells were cultured for 1 and 7 days; cells grown on the well surface in the absence of scaffolds represented the control condition. Prior to cytotoxicity assays, chitosan scaffolds were sterilized by serial incubation ( $2 \mathrm{~h}$ each) with $96 \%, 70 \%$, and $50 \%$ ethanol solutions in sterile PBS (v/v); after that, the scaffolds were washed three times for $10 \mathrm{~min}$ with sterile PBS before use. At the end of the assays, viability of cultured cells was assessed by the 3-(4,5-dimethylthiazol2-yl)-2,5-diphenyltetrazolium bromide (MTT) proliferation assay that measures mitochondrial activity. Briefly, after treatments, MTT solution $(5 \mathrm{mg} / \mathrm{mL}$ in sterile filtered PBS, $\mathrm{pH}$ 7.4) was added to each culture well of the 24-well plate, to reach the final concentration of $0.5 \mathrm{mg} \mathrm{MTT} / \mathrm{mL}$, and plates were incubated at $37^{\circ} \mathrm{C}$ for $4 \mathrm{~h}$. Then, after moving the scaffolds to collection tubes, the dark-blue formazan crystals produced by cell metabolization were solubilized by pipetting to induce cell lysis with a 2-propanol/1 $\mathrm{N} \mathrm{HCl}$ solution, and absorbances at $\lambda=550 \mathrm{~nm}$ were measured by a spectrophotometer. Data were normalized and reported as percent of the control condition (conventionally $100 \%$ proliferation) and were the mean $( \pm$ SEM) of 6 sample replicates for each treatment.

Cytocompatibility Assays on Human Mesenchymal Stem Cells (hMSCs). Cell viability assays were performed also on a human mesenchymal stem cell line (hMSC) obtained from Lonza (Milano, Italy). hMSCs were cultured in $75 \mathrm{~cm}^{2}$ cell culture flasks in Eagle's minimum essential medium alpha modification ( $\alpha$-MEM) supplemented with 10\% FBS $(\mathrm{v} / \mathrm{v})$, mixed antibiotic solution $(100 \mu \mathrm{g} / \mathrm{mL}$ streptomycin and $100 \mathrm{U} / \mathrm{mL}$ penicillin), and $2 \mathrm{mM}$ L-glutamine. hMSCs at passage 4 of propagation were used for all the experimental procedures, in a water-saturated atmosphere of $5 \% \mathrm{CO}_{2}$ and $95 \%$ air $\left(37^{\circ} \mathrm{C}\right)$. For the proliferation assays, $7 \times 10^{3}$ cells were seeded in triplicate onto the scaffolds (diameter: $9 \mathrm{~mm}$ ) in multiwell plates; at each time point, cells grown on the well surface in the absence of the scaffolds represented the control condition. Prior to seeding, scaffolds were sterilized by serial incubation ( $2 \mathrm{~h}$ each) with $96 \%, 70 \%$, and $50 \%$ ethanol solutions in sterile PBS; after that, the scaffolds were washed three times with sterile PBS. The cell proliferation was checked by the standard protocol of the Alamar Blue assay at 1 and 7 days of culture; for the hMSC cells, the Alamar Blue assay was chosen as it is suitable for evaluating the viability of particularly sensitive cell cultures when the number of seeded cells is lower than $5 \times 10^{4}$; based on previous work [29], the culture medium was changed every two days during the assays. The optical density was then measured with a spectrophotometer (Victor X3, PerkinElmer, Italy) at $\lambda=540$ and $\lambda=600 \mathrm{~nm}$. 


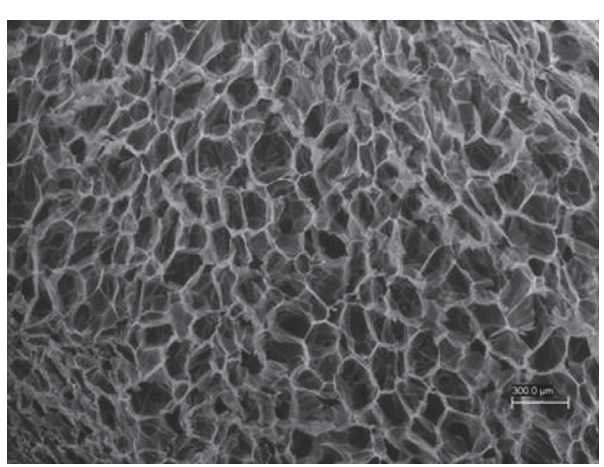

(a)

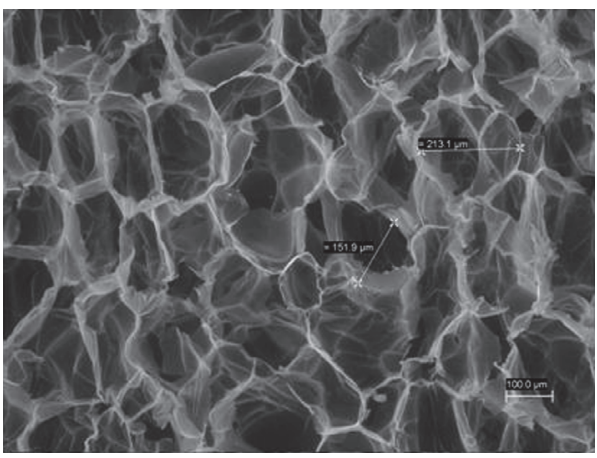

(c)

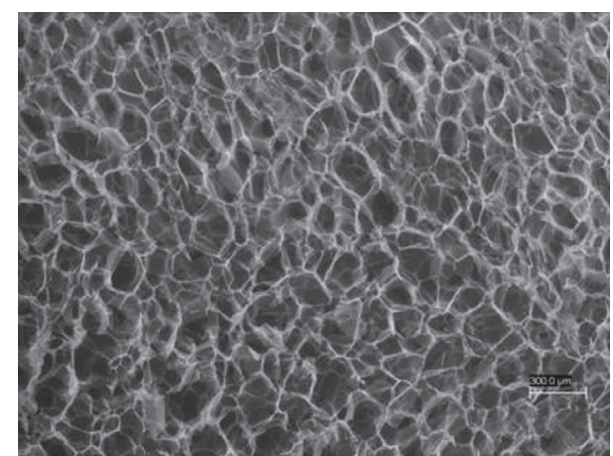

(b)

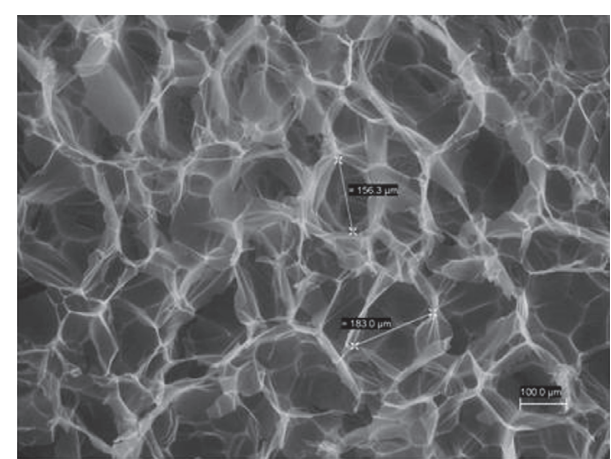

(d)

FIGURE 1: (a) GN1 CS scaffold at 100x magnification in transversal section; (b) GN2 CS scaffold at 100x magnification in transversal section; (c) GN1 CS scaffold at 250x magnification and pore size measurements in transversal section; (d) GN2 CS scaffold at 250x magnification and pore size measurements in transversal section.

\section{Results and Discussion}

3.1. Morphological Analysis. SEM images demonstrate that the freeze-drying method allows obtaining matrices with a highly interconnected porosity. In transversal sections, both samples, GN1 and GN2, show isotropic porosity and pore shape (Figures 1(a) and 1(b)). No relevant differences have been observed by the genipin content in the two scaffolds and no correlation has been found between the increasing of genipin content and porosity changes. Pore diameter of both scaffolds ranges from 150 to $200 \mathrm{~nm}$ in both samples (pore measurements in Figures 1(c) and 1(d)).

3.2. Degradation Study. To study the weight loss of GN1 and GN2 cross-linked CS scaffolds, in vitro degradation tests were carried out in PBS (GN1 and GN2 samples) and hydrolytic solution (GN1 and GN2 lyso samples) at $37^{\circ} \mathrm{C}$. In fact, it is well known that cells in culture produce metalloproteases and biomolecules that may alter the degradation kinetics of scaffolds. The weight loss for the samples is shown in Figure 2. It can be observed that the weight loss for the GN2 and GN2 lyso scaffolds proceeds slowly during the whole degradation period. After 28 days of incubation, both samples had lost about $33 \%$ of their initial mass. This is due to the high concentration of cross-linking degree. In fact, because of the introduction of cross-links, on average, more chains have to be cleaved before a degraded fragment can

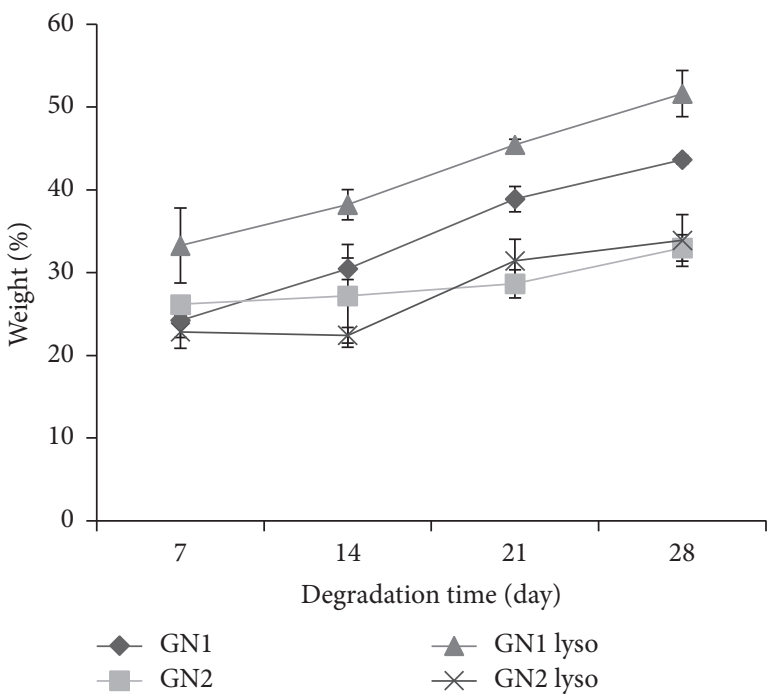

FIGURE 2: In vitro degradation of the genipin-CS scaffolds exposed to PBS and to the hydrolytic solution.

be solubilized. Moreover, the higher concentration of the cross-linking agent can increase the cross-link density of the scaffold and limit penetration of the enzyme into the specific sites of the polymer chains and the subsequent degradation. With the decreasing of cross-linking agent, the weight loss 


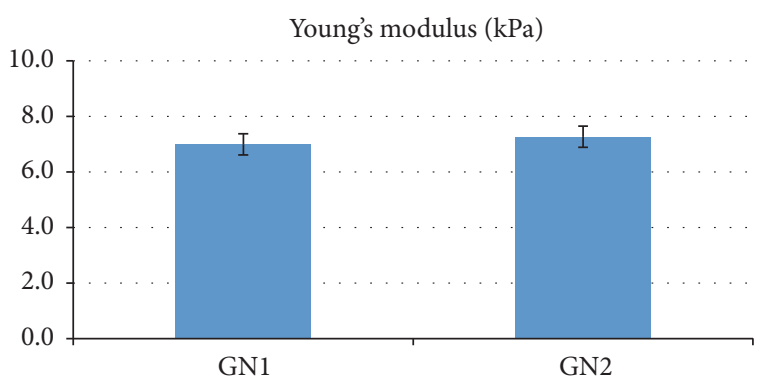

FIGURE 3: Average value and corresponding standard deviation value of the elastic modulus $\left(E_{\text {low }}\right)$ calculated by initial slope of the stress-strain curves obtained by compression tests.

of samples is increased. As reported in the plot, GN1 and GN1 lyso scaffolds exhibited gradual degradation until they reached $52 \%$ and $44 \%$ of the original mass after 28 days of experiment, respectively. When compared with GN2 and GN2 lyso, GN1 and GN1 lyso scaffolds exhibited a different trend of degradation kinetics: the weight loss increased much more quickly in the GN1 lyso samples, thanks to the easy penetrability of the lysozyme.

3.3. Mechanical Properties. The compression tests showed that as the cross-linking degree increases, no significant differences can be reported between GN1 and GN2 samples (Figure 3). Different authors reported a decrease of the elastic modulus above limit value in the genipin concentration. These systems are usually biological explants or polymeric systems treated with genipin [30,31]. Interestingly, in tests, GN2 scaffolds present the same modulus of GN1, even when the genipin concentration is double. In our systems, the presence of a structure with higher porosity, randomly oriented, leads to a structure able to react to compression loads. The effect of the chemical cross-link is negligible if compared to the macroscopic structure.

3.4. Contact Angle Analysis. Contact angles of different hydrogels compositions ((a) CS, (b) GN1, and (c) GN2) are shown in Figure 4. It could be seen that, with the increase of genipin content from $0 \%$ (a) to $3.75 \%$ (b) to $7.5 \%$ (c) w/w of dry chitosan, the contact angle of hydrogels surface significantly decreases from $101.75^{\circ}$ (a) to $79.455^{\circ}$ (b) and $72.485^{\circ}$ (c) $(P<0.05)$. There were no significant differences $(P>0.05)$ among contact angles of gels $(b)$ and $(c)$, indicating that the additional increase of genipin amount above 3.5\% w/w dry chitosan did not markedly affect the hydrophilicity of the gel surface. These results are in accordance with the study performed by Gao et al. [32].

\subsection{Fourier Transform Infrared (FTIR) Spectroscopy Analysis.} Figure 5 shows FTIR spectra recorded for pellets of genipin, a CS control, and CS-genipin complex.

The spectrum of unreacted genipin is characterized by three bands at 990,1080 , and $1620 \mathrm{~cm}^{-1}$, assigned to the $\mathrm{C}-\mathrm{H}$ ring out-of-plane bend, $\mathrm{C}-\mathrm{H}$ ring in-plane bend, and $\mathrm{C}=\mathrm{C}$ double bond ring stretch modes of the core of the

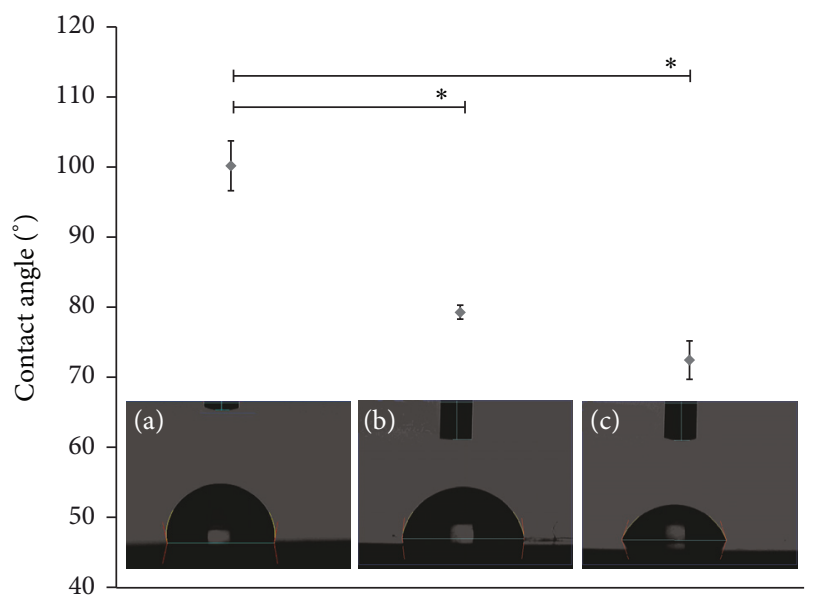

FIGURE 4: Comparison of contact angle on non-cross-liked CS and genipin cross-linked CS gels, GN1 and GN2 ((b) and (c), resp.); ${ }^{*} \mathrm{P}<$ 0.05 ; error bars represent mean value \pm standard deviation $(n=3)$.

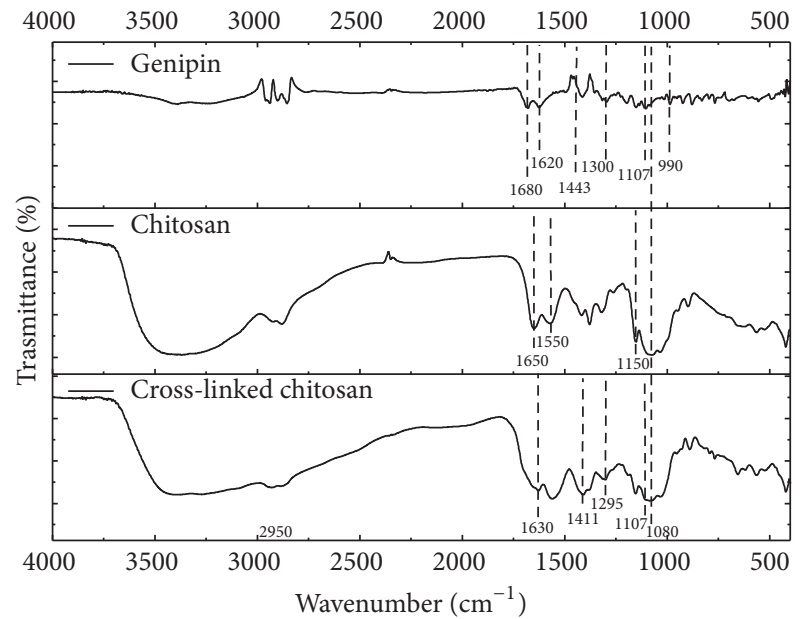

FIGURE 5: The FTIR spectra of genipin and CS before cross-linking with genipin and CS after cross-linking.

genipin molecule, respectively. The absorption at $1080 \mathrm{~cm}^{-1}$ may also include the $\mathrm{C}-\mathrm{O}$ stretch mode of the primary alcohol on the genipin molecule [33-35]. Additionally, the C-O-C asymmetric stretch and the $\mathrm{CH} 3$ bend of the methyl ester were observed at 1300 and $1443 \mathrm{~cm}^{-1}$, respectively; the crosslinked chitosan spectrum features these bands. Absorption at $1107 \mathrm{~cm}^{-1}$ is assigned to the vibrations of the cyclic ether.

The spectrum of chitosan is characterized by several absorption bands; the peaks at $1650 \mathrm{~cm}^{-1}$ and $1550 \mathrm{~cm}^{-1}$ were assigned to $\mathrm{C}=\mathrm{O}$ stretching in amide $\mathrm{I}$ and $\mathrm{N}-\mathrm{H}$ bending in amide II, respectively. The band at $1151 \mathrm{~cm}^{-1}$ was assigned to characteristic asymmetric stretching of C-O-C of polysaccharide structure [36].

After genipin cross-linking, the samples revealed additional peaks at 1295,1440 , and $1630 \mathrm{~cm}^{-1}$, which were assigned to the $\mathrm{C}-\mathrm{O}-\mathrm{C}$ asymmetric stretching and the $\mathrm{CH} 3$ bending of the methyl ester and $\mathrm{C}=\mathrm{C}$ ring stretching, respectively, in agreement with other FTIR studies on genipin 
treated biopolymers [37]. These features suggest that the carboxymethyl group of genipin reacted with the amino group of chitosan to form a secondary amide according to the literature [28].

The band at 1107 was assigned to the C-N stretch of the tertiary aromatic amine [33] of the cross-linked genipin nitrogen iridoid that is bound covalently to the chitosan. Furthermore, the band at $1104 \mathrm{~cm}^{-1}$ in the cross-linked spectrum is significantly stronger than the corresponding band in the spectrum of pure genipin (relative to the band at $1080 \mathrm{~cm}^{-1}$ ), suggesting that this absorption band is mostly associated with modes formed as a result of crosslinking.

3.6. Biocompatibility Evaluation Assays on Human-Derived Cell Lines. Basic biocompatibility of the GN1- and GN2type structures was preliminarily evaluated by assaying proliferation of cultured human-derived cell lines after 1 and 7 days of culture, chosen as time points for assessing the short-term proliferative behavior of cells. To this aim, the immortalized MG63 human osteoblast-like cells and human mesenchymal stem cells (hMSCs) were adopted as assessed models for bone tissue engineering [32, 33, 38]. Results from MTT assays on MG63 seeded onto GN1 and GN2 scaffolds showed no significant effects on metabolic activity and proliferation of cells with respect to the control cells grown on standard culture plates. Additionally, results also showed that there was no statistically different proliferation between GN1 and GN2 scaffolds after 1 as well as 7 days (Figure 6). Moreover, the slight nonsignificant decrease observed at day 1 is shown to be recovered at day 7; this aspect suggests a reduced impact of the material at day 1 , when seeded cells are entering the high-sensitive phase of the first doubling of their number. Also, data suggest that CS scaffolds show good permissiveness towards MG63 cell proliferation and that the effects of different cross-linking between GN1 and GN2 can be contained and ad hoc modulated within a range of optimal viability. On this basis, cell viability was also assessed on the hMSCs by the Alamar Blue assay, in order to evaluate the effect of materials on undifferentiated cells (hMSCs). As shown in Figure 7, reduced proliferation (50 and $40 \%$ for GN1 and GN2, resp.) was detected at day 1 with respect to control cells (100\%); however, recovery of viability was detected at day 7 (up to 73 and $70 \%$ for GN1 and GN2, resp.). This evidence of recovery is indicative of the ability of cells to prosecute the physiological proliferative pathways, consequent to adhesion. On the other hand, the reduced proliferation at day 1 could be due to the cell typespecific sensitiveness of the hMSCs with respect to MG63. This different initial interaction of the two cell lines with the scaffolds could give interesting hints on the versatility of the materials, thus deserving to be further investigated, for example, in terms of the differential gene expression induced by the cell-material dynamic contact. In terms of differences between GN1 and GN2, it can be noticed that as previously observed for the MG63 cells, no significant deviation was detected for the hMSC cells.

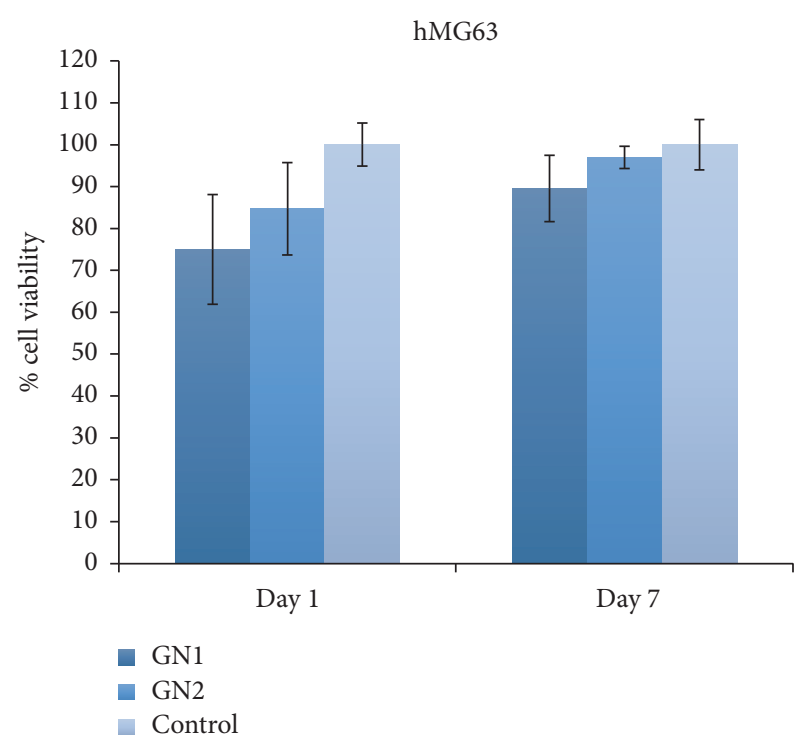

Figure 6: Cell proliferation evaluation by MTT assays on MG63 cells grown in the presence of GN1 and GN2 CS scaffolds after 1 and 7 days of culture. Data are expressed as percent viability $( \pm$ SEM) with respect to the control.

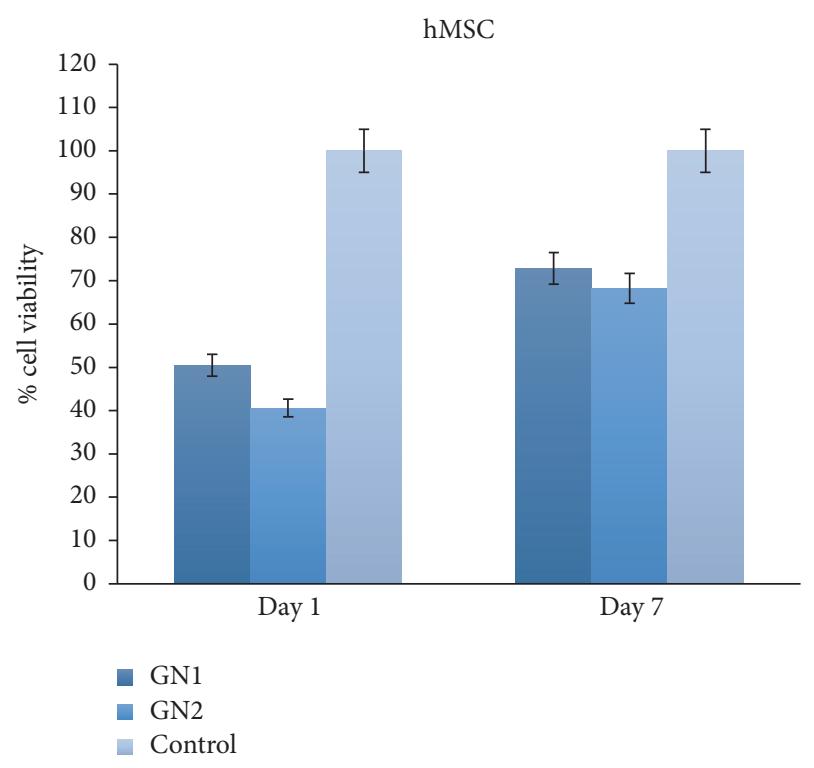

FIgURE 7: Cell proliferation assays by Alamar Blue on hMSC cells grown in the presence of GN1 and GN2 CS (at 1 and 7 days of culture). Data are reported as percent viability ( \pm SEM) with respect to control and derive from the average of 6 replicate values $(n=6)$.

\section{Conclusions}

Overall, this study describes genipin cross-linked chitosan scaffolds as suitable systems for bone tissue engineering. Different genipin concentrations effectively change degradation profiles, but not the mechanical properties of the cross-linked chitosan scaffold structures. At the same time, there is no effect on morphology and porosity. This suggests that the effect on the elastic modulus is generally influenced 
principally by morphological modifications and not by the chemically modified structure due to the effect of the crosslinking reaction. In addition, the basic biocompatibility evaluation indicates that the cell-material interactions do not induce significant cytotoxic effects, thus well supporting cell growth over time. Interestingly, the difference in crosslinking of GN1 versus GN2, that is, the difference in genipin concentrations, does not have significantly dissimilar impact on proliferation of both cell lines under investigation, while it has a pivotal impact on the structural and mechanical properties of the scaffolds. On this basis, genipin concentration may represent a modular parameter functional to the differential cell-specific targeting ability of a putative bone-mimicking scaffold.

\section{Conflicts of Interest}

The authors declare no conflicts of interest.

\section{Authors' Contributions}

Christian Demitri and Amilcare Barca conceived the study and designed the experiments. Simona Dimida performed the experiments, analyzed the data, and wrote the paper. Nadia Cancelli performed the mechanical tests. Maria Grazia Raucci performed the biological analyses with hMSC. Simona Dimida and Amilcare Barca contributed equally to this work.

\section{Acknowledgments}

The authors wish to thank Donato Cannoletta from the microscopy lab of the Department of Engineering for Innovation, University of Salento, for the SEM analyses.

\section{References}

[1] A. Oryan, S. Alidadi, and A. Moshiri, "Current concerns regarding healing of bone defects," Hard Tissue, vol. 2, no. 2, article 13, 2013.

[2] W. R. Moore, S. E. Graves, and G. I. Bain, "Synthetic bone graft substitutes," ANZ Journal of Surgery, vol. 71, no. 6, pp. 354-361, 2001.

[3] C. Laurencin, Y. Khan, and S. F. El-Amin, "Bone graft substitutes," Expert Review of Medical Devices, vol. 3, no. 1, pp. 49-57, 2006.

[4] C. R. Perry, "Bone repair techniques, bone graft, and bone graft substitutes," Clinical Orthopaedics and Related Research, no. 360, pp. 71-86, 1999.

[5] F. Scalera, C. Esposito Corcione, F. Montagna, A. Sannino, and A. Maffezzoli, "Development and characterization of UV curable epoxy/hydroxyapatite suspensions for stereolithography applied to bone tissue engineering," Ceramics International, vol. 40, no. 10, pp. 15455-15462, 2014.

[6] S. Ishack, A. Mediero, T. Wilder, J. L. Ricci, and B. N. Cronstein, "Bone regeneration in critical bone defects using threedimensionally printed $\beta$-tricalcium phosphate/hydroxyapatite scaffolds is enhanced by coating scaffolds with either dipyridamole or BMP-2," Journal of Biomedical Materials Research Part B: Applied Biomaterials, vol. 105, no. 2, pp. 366-375, 2017.
[7] K.-W. Park, Y.-P. Yun, S. E. Kim, and H.-R. Song, "The effect of alendronate loaded biphasic calcium phosphate scaffolds on bone regeneration in a rat tibial defect model," International Journal of Molecular Sciences, vol. 16, no. 11, pp. 26738-26753, 2015.

[8] J. Zhang, W. Liu, O. Gauthier et al., "A simple and effective approach to prepare injectable macroporous calcium phosphate cement for bone repair: syringe-foaming using a viscous hydrophilic polymeric solution," Acta Biomaterialia, vol. 31, pp. 326-338, 2016.

[9] E. Boccardi, A. Philippart, J. A. Juhasz-Bortuzzo et al., "Uniform surface modification of 3D Bioglass ${ }^{\circledR}$-based scaffolds with mesoporous silica particles (MCM-41) for enhancing drug delivery capability," Frontiers in Bioengineering and Biotechnology, vol. 3, article 177, 2015.

[10] C. Demitri, M. G. Raucci, A. Giuri et al., "Cellulose-based porous scaffold for bone tissue engineering applications: assessment of hMSC proliferation and differentiation," Journal of Biomedical Materials Research Part A, vol. 104, no. 3, pp. 726733, 2016

[11] M. G. Raucci, M. A. Alvarez-Perez, C. Demitri, A. Sannino, and L. Ambrosio, "Proliferation and osteoblastic differentiation of hMSCS on cellulose-based hydrogels," Journal of Applied Biomaterials and Functional Materials, vol. 10, no. 3, pp. 302307, 2012.

[12] C. Demitri, A. Giuri, M. G. Raucci et al., "Preparation and characterization of cellulose-based foams via microwave curing," Interface Focus, vol. 4, no. 1, Article ID 20130053, 8 pages, 2014.

[13] M. G. Raucci, M. A. Alvarez-Perez, C. Demitri et al., "Effect of citric acid crosslinking cellulose-based hydrogels on osteogenic differentiation," Journal of Biomedical Materials Research. Part A, vol. 103, no. 6, pp. 2045-2056, 2015.

[14] L. L. Hench and J. Wilson, "Surface-active biomaterials," Science, vol. 226, no. 4675, pp. 630-636, 1984.

[15] N. Rochet, T. Balaguer, F. Boukhechba et al., "Differentiation and activity of human preosteoclasts on chitosan enriched calcium phosphate cement," Biomaterials, vol. 30, no. 26, pp. 4260-4267, 2009.

[16] M. N. V. Ravi Kumar, "A review of chitin and chitosan applications," Reactive and Functional Polymers, vol. 46, no. 1, pp. 1-27, 2000.

[17] J. Nilsen-Nygaard, S. P. Strand, K. M. Vårum, K. I. Draget, and C. T. Nordgård, "Chitosan: gels and interfacial properties," Polymers, vol. 7, no. 3, pp. 552-579, 2015.

[18] R. Muzzarelli, "Chitosan," in Natural Chelating Polymers, R. Muzzarelli, Ed., pp. 144-176, Pergamon Press, Oxford, UK, 1973.

[19] N. Van Toan, C.-H. Ng, K. N. Aye, T. S. Trang, and W. F. Stevens, "Production of high-quality chitin and chitosan from preconditioned shrimp shells," Journal of Chemical Technology and Biotechnology, vol. 81, no. 7, pp. 1113-1118, 2006.

[20] N. Bhattarai, J. Gunn, and M. Zhang, "Chitosan-based hydrogels for controlled, localized drug delivery," Advanced Drug Delivery Reviews, vol. 62, no. 1, pp. 83-99, 2010.

[21] D. M. Ornoff, Y. Wang, A. Proctor, A. S. Shah, and N. L. Allbritton, "Co-fabrication of chitosan and epoxy photoresist to form microwell arrays with permeable hydrogel bottoms," Biomaterials, vol. 74, pp. 77-88, 2016.

[22] L. Xu, Y.-A. Huang, Q.-J. Zhu, and C. Ye, "Chitosan in molecularly-imprinted polymers: current and future prospects," International Journal of Molecular Sciences, vol. 16, no. 8, pp. 18328-18347, 2015. 
[23] P. Ghosh, A. P. Rameshbabu, D. Das et al., "Covalent cross-links in polyampholytic chitosan fibers enhances bone regeneration in a rabbit model," Colloids and Surfaces B: Biointerfaces, vol. 125, pp. 160-169, 2015.

[24] X. Shu and K. Zhu, "Controlled drug release properties of ionically cross-linked chitosan beads: the influence of anion structure," International Journal of Pharmaceutics, vol. 233, no. 1-2, pp. 217-225, 2002.

[25] E.-C. Shen, C. Wang, E. Fu, C.-Y. Chiang, T.-T. Chen, and S. Nieh, "Tetracycline release from tripolyphosphate-chitosan cross-linked sponge: a preliminary in vitro study," Journal of Periodontal Research, vol. 43, no. 6, pp. 642-648, 2008.

[26] R. A. A. Muzzarelli, "Genipin-crosslinked chitosan hydrogels as biomedical and pharmaceutical aids," Carbohydrate Polymers, vol. 77, no. 1, pp. 1-9, 2009.

[27] H. W. Sung, D. Huang, W. Chang, R. Huang, and J. C. $\mathrm{Hsu}$, "Evaluation of gelatin hydrogel crosslinked with various crosslinking agents as bioadhesives: in vitro study," Journal of Biomedical Materials Research, vol. 46, no. 4, pp. 520-530, 1999.

[28] S. Dimida, C. Demitri, V. M. De Benedictis, F. Scalera, F. Gervaso, and A. Sannino, "Genipin-cross-linked chitosan-based hydrogels: reaction kinetics and structure-related characteristics," Journal of Applied Polymer Science, vol. 132, no. 28, Article ID 42256, 2015.

[29] S. K. Bopp and T. Lettieri, "Comparison of four different colorimetric and fluorometric cytotoxicity assays in a zebrafish liver cell line," BMC Pharmacology, vol. 8, article 8, 2008.

[30] G. Fessel, J. Cadby, S. Wunderli, R. Van Weeren, and J. G. Snedeker, "Dose- and time-dependent effects of genipin crosslinking on cell viability and tissue mechanics-toward clinical application for tendon repair," Acta Biomaterialia, vol. 10, no. 5, pp. 1897-1906, 2014.

[31] M.-C. Chen, C.-T. Liu, H.-W. Tsai, W.-Y. Lai, Y. Chang, and H.W. Sung, "Mechanical properties, drug eluting characteristics and in vivo performance of a genipin-crosslinked chitosan polymeric stent," Biomaterials, vol. 30 , no. 29 , pp. 5560-5571, 2009.

[32] L. Gao, H. Gan, Z. Meng et al., "Effects of genipin crosslinking of chitosan hydrogels on cellular adhesion and viability," Colloids and Surfaces B: Biointerfaces, vol. 117, pp. 398-405, 2014.

[33] G. Socrates, Infrared and Raman Characteristic Group Frequencies: Tables and Charts, Wiley, New York, NY, USA, 2004.

[34] M. Arteche Pujana, L. Pérez-Álvarez, L. C. Cesteros Iturbe, and I. Katime, "Biodegradable chitosan nanogels crosslinked with genipin," Carbohydrate Polymers, vol. 94, no. 2, pp. 836-842, 2013.

[35] R. Touyama, Y. Takeda, K. Inoue et al., "Studies on the blue pigments produced from genipin and methylamine. I. Structures of the brownish-red pigments, intermediates leading to the blue pigments," Chemical and Pharmaceutical Bulletin, vol. 42 , no. 3, pp. 668-673, 1994.

[36] J.-Y. Lai, Y.-T. Li, and T.-P. Wang, "In vitro response of retinal pigment epithelial cells exposed to chitosan materials prepared with different cross-linkers," International Journal of Molecular Sciences, vol. 11, no. 12, pp. 5256-5272, 2010.

[37] H. G. Sundararaghavan, G. A. Monteiro, N. A. Lapin, Y. J. Chabal, J. R. Miksan, and D. I. Shreiber, "Genipin-induced changes in collagen gels: correlation of mechanical properties to fluorescence," Journal of Biomedical Materials Research Part A, vol. 87, no. 2, pp. 308-320, 2008.
[38] E. M. Czekanska, M. J. Stoddart, R. G. Richards, and J. S. Hayes, "In search of an osteoblast cell model for in vitro research," European Cells and Materials, vol. 24, pp. 1-17, 2012. 

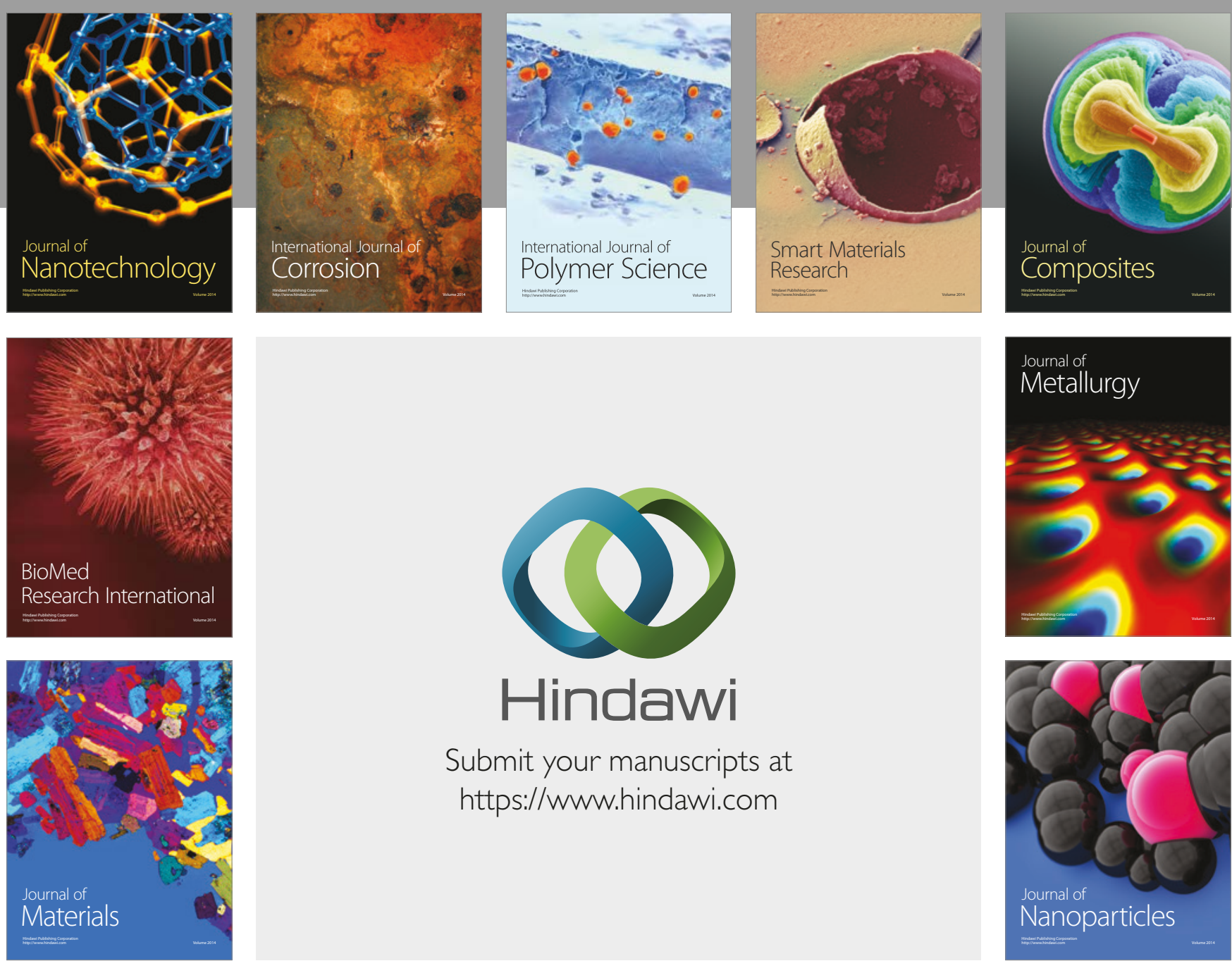

\section{Hindawi}

Submit your manuscripts at

https://www.hindawi.com
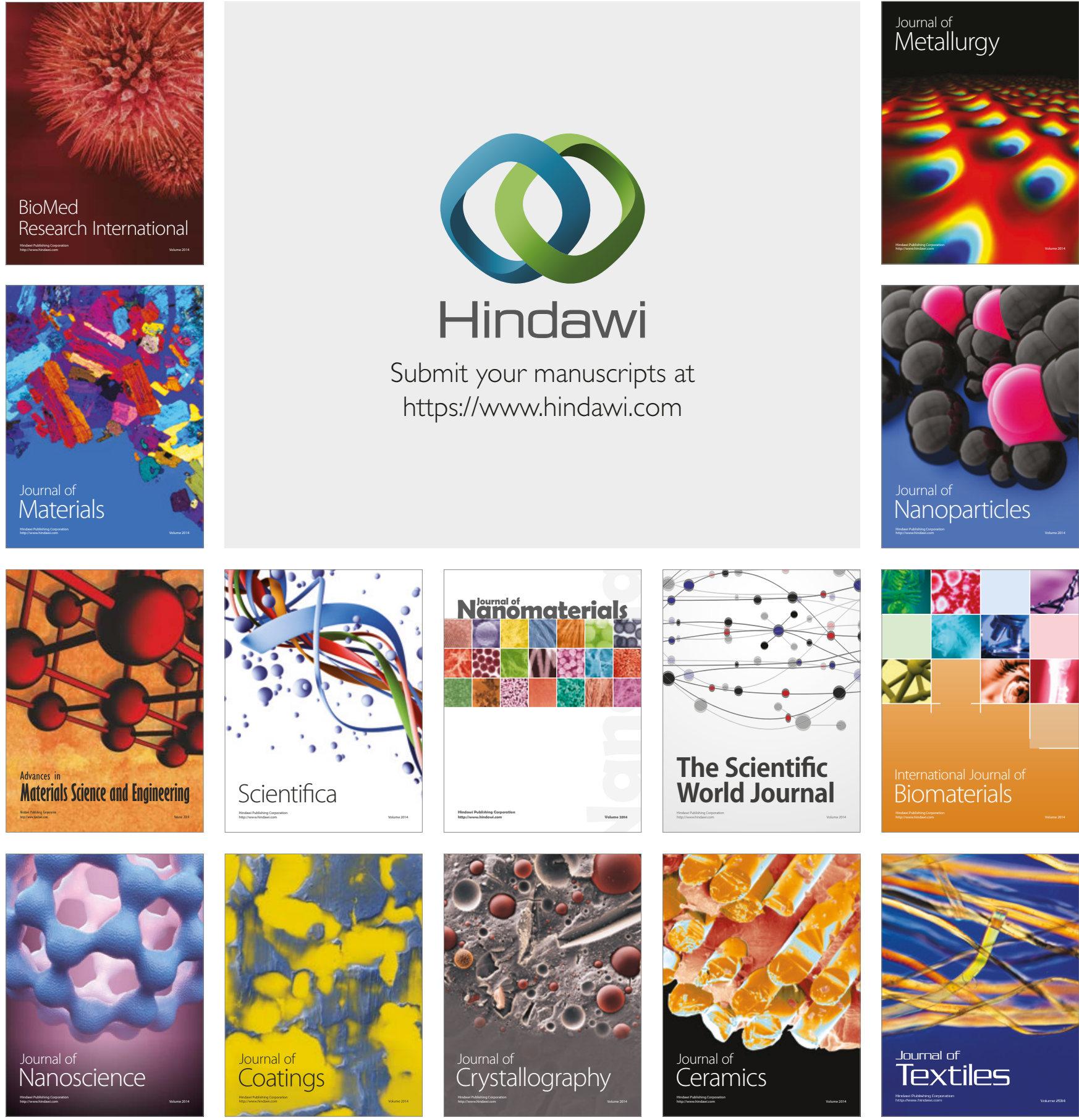

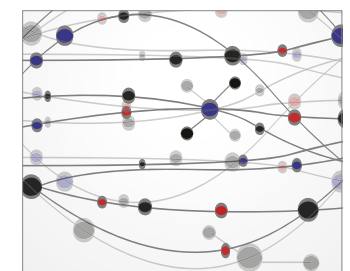

The Scientific World Journal
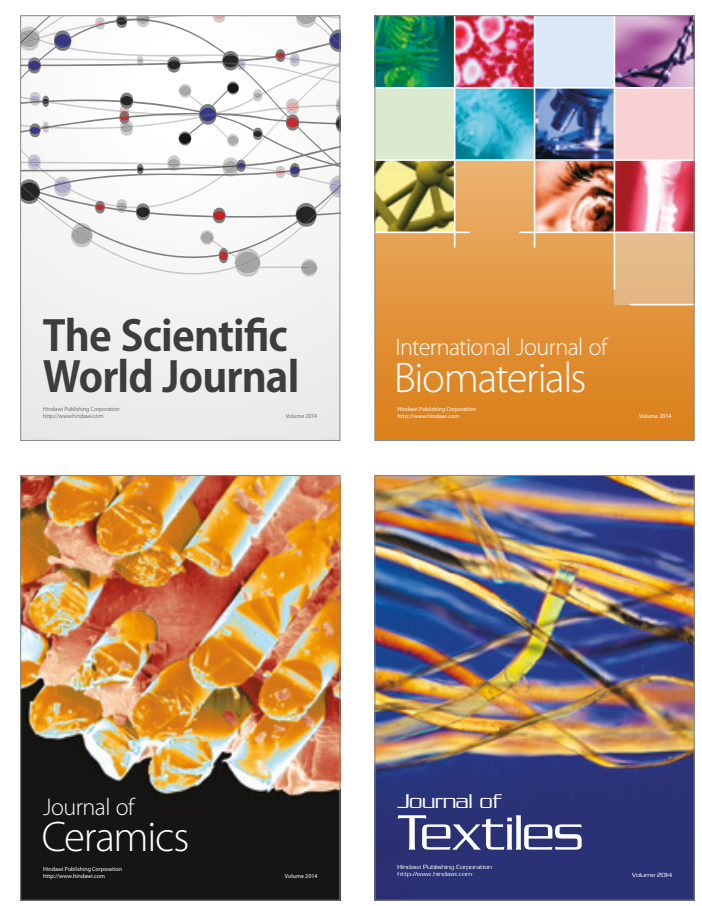